\section{ANEURISM OF THE ARTERIA} INNOMINATA.

\section{LIGATURE OF THE COMMON CAROTID AND SUBCLAVIAN} ARTERIES SIMULTANEOUSLY.

BY FREDERICK ENSOR, M.R.C.S., SURGEON TO PORT ELIZABETH HOSPITAL, SOUTH AFRICA.

THE treatment of aneurism of the arteria innominata by proximal ligature, I believe, has been so disastrous as to be rejected, giving no hope of success. That by distal ligature of the common carotid and subclavian arteries has, in the rare cases recorded, presented results of a more encouraging and hopeful character; and I think that, especially, the case of Mr. Christopher Heath, the preparation of which I saw last year in the Museum of the Royal College of Surgeons, gives a good precedent, and warrants the undertaking of a similar operation, guarded by extreme care in diagnosis, and when, from the condition of the patient, there seems a fair chance of good being done.

In the case I now proceed to detail, I proposed to myself two objects-first, possible cure of the aneurism, or at least such modification of the sac as would retard its spreading, and so prolong life; and, secondly, if I failed to arrest it altogether, $I$ thought $I$ might arrest its progress anteriorly, and so prevent that erosion and tension of the chest-wall which is the cause of such miserable suffering in cases of aneurism of the arch and its large offsets. If this failed to be effected, at least there would be a probability of the sac giving way elsewhere, and I should cause the end to be an euthanasia in comparison to that which I have witnessed in cases of aneurism which have been left to nature. From experience in this hospital, I may say that aneurism, especially of the aorta, is by no means an uncommon cause of admission. I think the greatest suffering I ever saw a human being undergo was in a case of aneurism of the arch, which occurred a few years ago in the person of the patient whose photo I enclose, where it will be noticed that the whole of the chest-wall is pushed out and thinned to an extreme degree. The amount of suffering this involved was so great that I thought at the time any action would be justifiable which gave the patient even a remote chance of alleviating such burning, gnawing pain. On more than one occasion this poor man contemplated self-destruction to escape from his torture.

At the risk of being considered tedious and taking up too much of your valuable space, I report the present case in detail, for I felt that in conducting so grave an experiment I should have been glad to have been able to refer to the daily progress of a similar case.

Wideman Stuurman, a bastard Kaffir Hottentot, was admitted into the Provincial Hospital, Port Elizabeth, on the 25th May, 1874. He was a man of medium height, strongly built, but lean; arcus senilis complete in both eyes; looked about fifty years of age; confessed to having drunk hard. His area of liver dulness, on percussion, was too small dulness and medium moist sounds over bases of lungs; heart's apex beating very visibly below the nipple; dulness over middle of sternum, and-a bruit increasing as you passed across the chest to the right, and most pronounced over the right sterno-clavicular articulation. Pulse 72 . I put the man on a spare meat diet, and gave him three minims of tincture of aconite night and morning.

May 29th.-The aconite was used to lower the pulse, but proved too depressing, and although its influence on the impulse was marked, I was obliged to omit it.

July 1st.-Pulsation of tumour more pronounced, and the finger is sensihly raised on placing it at root of the neck above the sterno-clavicular articulation. Man is thinning; pain in the chest more severe; sensitiveness of skin over the whole body; he flinches when touched.

August 8th.-Pulse 82. He has been using fifteen minims of chlorodyne three or four times a day for irritable bowels, which is now relieved. Beating at heart's apex not so marked; the upper border of the sternum is getting prominent, and a marked see-saw sound is heard there; veins of neck enlarging, and some odema of left arm; objects to being kept in bed, and getting restless from confinement.

26th. - Pulse 83. Working a little at weeding in the garden; feels very giddy when stooping; appetite good no pain on swallowing; lies without distress on either side; pulse very marked over right sterno-clavicular articulation; heart's apex beats two inches and a half below the nipple.

Sept. 4th.-Feels more pain in chest, and the impulse at the upper part of the chest more evident to sight and touch. The man is getting impatient, and says be will go away if I will not do something for him. The natives generally fear physic; they fancy it savours too much of witchdoctoring. But they " go bravely to surgery." As I think, from careful frequent observation, that I have to deal with a somewhat dilated arch of the aorta, with a second pouch or dilatation in the arteria innominata, I think, to give the man a chance of relief, and perhaps prolong life, I will venture an operation.

8th.-In the presence of $\mathrm{my}$ colleagues, M. Dunstaulle, Le Scour, Hall, and two other visiting medical men, I proceeded to place a ligature on the common carotid artery just immediately below the omohyoid muscle, and on the subclavian as it crosses the first rib. At 8 A m. the man was allowed a little coffee and toast. At 11 A.M., immediately before the operation, I gave him an ounce and a half of brandy. His pulse just before giving chloroform was 72 . Taking the cricoid cartilage as a centre, I made an incision as nearly as possible an inch and a balf on each side of it, on the border of the sterno-mastoid muscle. The anterior jugular, prominent and full, had to be carefully avoided, and also a network of veins which presented themselves low down in the wound. Anxiety to prevent the obscuring of parts by venous blood caused me to be longer than I anticipated in opening the sheath over the artery just at the lower edge of the omohyoid muscle. The artery being cleared by the director, I had no difficulty in passing the needle carrying a silk ligature under it. The cutting off of so large a supply of blood from the brain had no perceptible effect on the patient. Without allowing him to emerge from the effect of the chloroform, which be took kindly, there being no embarrassment of beart or lungs, I pulled his right arm well down over the side, and fastened it by the wrist to the leg of the table. This effectually kept the shoulder down without an assistant being in the way. Making the usual incision from the anterior border of the trapezius to the edge of the sterno-mastoid muscle, I cut a small artery, which was the only one which was severed in either operation; its jet stopped without a ligature; the little venous hæmorrhage, which temporarily obscured the outer edge of the wound, was quickly sponged up, and ceased to trouble. The external jugular, large and gorged by transverse veins joining it, came into view as the retracted skin sprang into its place above the clavicle. The deep fascia was freely opened on a director, the veins held to the inner side by a retractor. The finger, passed into the wound, came at once against the border of the scalenus anticus and its insertion into the tubercle of the first rib, and the subclavian artery felt distinctly throbbing under the finger. Freeing its communication with the brachial nerve and loose tissue all around, the needle, armed as before, was without any difficulty passed under the vessel, the silk tied, shutting up the radial pulse. Both wounds were next closed with wire suture, and dressed with carbolicacid lotion and oiled silk.

The ligature of the sublavian was accomplished in a few minutes, and seemed to me a much easier affair than that of the carotid. In the present instance the carotid lay deep; on account of the shortness of the man's neck and the bulk of the sterno-mastoid muscle. The engorged venous network was also a cause of more than ordinary care and consequent time spent in manipulation.

Before removal to his bed the man spoke intelligently, and his pulse was 82 . In the evening, feeling restless, he was ordered thirty minims of solution of morphia.

9th.-Pulse: morning, 84; evening, 88. Pulsation less marked at upper border of sternum. Says he feels less pain in the chest. Bowels too free. Diet: Mill and tea and toast. No disturbance of the respiration. Ordered one drachm of solution of morphia at kedtime.

10th.-Slept well. A little blood in the motions. Ordered chalk mixture with chlorodyne, twenty minims. Pulse: morning, 94; evening, 90. Respiration natural ; skin moist 
and warm. Takes tea and milk, and minced meat; the latter swallowed without difficulty, and enjoyed. To omit night draught.

11th.-Palse : morning, 98; evening, 105. Has had three motions. Impulse over sternum decidedly less. Respiration natural. Wounds doing well. Diet: tea and milk, and minced meat, ten ounces. Repeat chlorodyne and chalk mixture twice a day.

12th. - Pulse : morning, 96; evening, 98. Complains of pain at the lower part of the sternum and on the right side. Pulsation very marked at and above the right sternoclavicular articulation; heaving of upper part of sternum less; bruit less marked. Ordered two minims of tincture of aconite and fifteen minims of chlorodyne every six hours. Diet the same.

13th.-Pulse: morning, 78; evening, 76. Purged four times. Skin moist and cool. Respiration natural. Pulsation over right sterno-clavicular joint less. Bruit much less. Pulse drops one now and then. Wounds discharging healthy pus.

14,th.-Bowels quiet. Pulse : morning, 84, erening, 89 ; intermits now and then. Skin very moist and soft. Aconite seems to depress him too much. Ordered nitric acid and bark, and two eggs, in addition to his meat and tea. Impulse very feeble over sternum. Bruit very slight.

15th.-Pulse: morning, 20 ; evening 89 . The acid and bark disagrees. Returned to the chlorodyne (twenty-five minims) and infusion of gentian three times a day.

16th.-Pulse: morning, 88; evening, 80. Impulse at sterno-clavicular articulation much reduced. Heart's apex striking with force at point before mentioned. Voice clear. No disturbance of respiration. Add one minim of tincture of aconite to each dose of mixture.

17th.-Pulse: morning, 78; evening, 85. Carotid wound nearly healed. Suppuration moderate at subclavian. Sleeps much. Omit five minims of chlorodyne; continue the aconite.

18th.-Pulse: morning, 84; evening, 85. Two sutures removed from the outer angle of the subclavian wound, from which pus comes freely. Diet and medicine the same. 19th.-Pulse : morning, 76 ; evening, 82 .

20th-Pulse: morning, 81; evening, 86 .

21st.-Pulse : morning, 74; evening, 76. Carotid wound nearly healed. Suppuration free from subclavian. Wounds dressed with balsam of copaiba. Diet and medicine as before. 22nd.-Pulse: morning, 80 ; evening 78. All the sutures removed from the wounds. A little pus exudes at exit of carotid ligature.

23rd.-Pulse : morning, 80; evening, 87. Piles trouble him.

24th.-Pulse: morning, 80 ; evening, 88.

25th.-Pulse : morning, 88; evening, 78. Ordered twenty minims of chlorodyne, ten minims of tincture of chinchona, and one minim and a half of aconite three times a day, and compound ointment of galls for piles.

26 th.-Pulse 76 at 12 o' clock.

27th.-Pulse 80 at 12 o'clock. Pulsation at upper part of sternum less. Makes no complaint of pain in the chest. 28 th.-Pukse 78.

29th.-Pulse 84. Two minims of tincture of aconite three times a day.

30th.-Pulse 74. A little more suppuration from carotid wound. The radial pulse first felt to-day. Heart's action evidently controlled by the aconite.

Oct. 1st.-Pulse 78. The subclavian ligature came away

this morning. Pain in carotid wound.

2nd.-Pulse 82. Respiration quiet. Subclavian wound healing well. Pus exudes from carotid wound on pressure or on swallowing.

3rd.-Pulse 84. Pulsation rather marked in the carotid. Complains of burning pain in line of wound. Pulse distinct in radial artery.

4.th.-Pulse 82. Pulsation heaving in the carotid. Suspended a split-shot weighing twenty grains to the carotid ligature, with view of exerting gentle and persistent traction.

5 th.-Pulse 80 .

6th.-Pulse 80. The nurse found the carotid ligature with the shot lying on his chest on dressing wound in the morning. Subelavian wound nearly healed.

7th.-Pulse 70. A little pus from carotid wound. Bowels quiet. To take the medicine but twice a day.
8th.-Pulse 84.

9th.-Pulse 76. Subclavian wound nearly skinned over.

He sleeps well ; complains but little of pain in the chest 10th.-Pulse 74. One minim of tincture of aconite three times a day.

11th.-Pulse 76. Allowed to dress and move gently bout.

12th.-Pulse 80. Bruit and impulse marked at upper part of sternum. Complains of pain on swallowing. Dulness on percussion in right suprascapular region, with moist sounds. Percussion clear at bases of both lungs. Pulse intermits now and then. Two minims of tincture of aconite twice a day.

13th.-Pulse 72. Had the mixture three times instead of twice. Pulse intermits and is feeble. Says, on question, that he has no pain in chest except an occasional passing pang, but has pain at upper part of the right lung behind.

14th.-Palse intermits frequently, and is very feeble. To take the medicine once a day only.

15th.-Sleeps, and has good appetite, but finds a difficulty in swallowing solids; mucus collects in windpipe and bronchi. Ordered porridge and milk instead of meat.

16th.-Pulse 80 ; no intermission. Medicine the same.

17th.-Pulse 80 ; no intermission. A direct bruit is heard over the sterno-clavicular articulation, and the pulsation is marked above the clavicle and across the lower part of the neck. Sleeps fairly and is dressed. Does not complain of any pain in the chest.

18th.-Pulse 76. Good deal of pain in the ear and side of head, which prevented sleep. Ordered fifteen grains of chloral hydrate at night. Noticed to-day that the right pupil was contracted to half the size of the left.

19th.-Slept well after the night draught. Pulse 76. To leave off the aconite, and take the chloral at night. Subclavian wound bealed. A little stain of pus on lint over the lower edge of the carotid wound.

20th.-Pulse 86 ; intermits occasionally. To resume the aconite once a day.

Thus far, I think, the operation may be considered to have done good. The patient has not so much pain as he had at the end of August. He makes no complaint of pain in the chest; his respiration is quiet; he can swallow semifluid food without pain or difficulty. The upper border of sternum and right sterno-clavicular articulation is not more prominent. The bruit is softer; the impulse is less. On the other hand, I think the tumour is rising in the neck, and he has some symptoma of pressure on the oesophagus and larynx. In the conduct of the case it is worthy of note how the aconite commanded the heart's impulse. The man sleeps well, enjoys his food, is in no particular distress, and is able to go about gently, but cannot make any exertion without embarrassment of the breathing.

I shall endeavour to keep him under observation, and the ultimate result shall, if desired, be made known in the pages of The Lancet.

\section{CASE OF UNUSUALLY RAPID PULSE;} TREATMENT BY DIGITALIS.

\section{BY JAMES BRISBANE, M.D.}

Mr. S_, an old patient of mine, sent for me on the 30th of June last. I found him suffering from acute rheumatism. He has chronic disease of the mitral valves, which, according to his own statement, is congenital. At all events it has been in existence ever since I have known him, now nearly six years. Nearly all the joints are affected, particularly the knees and unkles.

July 1st.-A decided friction-sound heard to-day, in addition to the chronic bruit.

2nd.-Alarmingly ill ; friction-sound very extensive, and the area of dulness over heart about double that of its normal extent. I requested a consultation with Dr. Handfield Jones, who saw him with me in the evening. From the feeble condition of the pulse Dr. Jones suggested that bark and ammonia should be administered. A blister was likewise applied over the heart. The patient recovered satisfactorily, and by the 20th of July he was able to go to 